\title{
AFM Imaging of RGD Presenting Synthetic Extracellular Matrix Using Gold Nanoparticles
}

\author{
Susan X. Hsiong, Peter H. Cooke, Hyun-Joon Kong, Marshall L. Fishman, \\ Maria Ericsson, David J. Mooney*
}

Several high-resolution imaging techniques such as FESEM, TEM and AFM are compared with respect to their application on alginate hydrogels, a widely used polysaccharide biomaterial. A new AFM method applicable to RGD peptides covalently conjugated to alginate hydrogels is described. High-resolution images of RGD adhesion ligand distribution were obtained by labeling biotinylated RGD peptides with streptavidin-labeled gold nanoparticles. This method may broadly provide a useful tool for SECM characterization and design for tissue regeneration strategies.

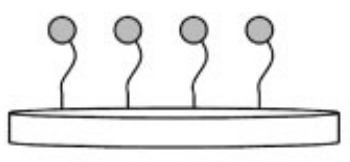

RGD modified alginate hydrogel

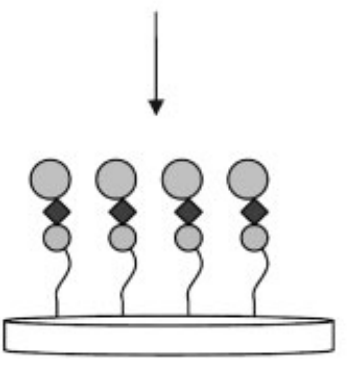

$\mathrm{G}_{4}$ RGDASSK

RGD-Biotin Peptide

Streptavidin conjugated gold nanospheres
S. X. Hsiong

Department of Chemical Engineering, University of Michigan, Ann Arbor, MI, USA

S. X. Hsiong, D. J. Mooney

School of Engineering and Applied Sciences, Engineering Science Lab, Harvard University SEAS, 325 Pierce Hall Cambridge, MA 02138, USA

Fax: +1 617496 5264; E-mail: mooneyd@seas.harvard.edu P. H. Cooke, M. L. Fishman

Eastern Regional Research Center, Agricultural Research Service, U.S. Department of Agriculture, Wyndmoor, PA, USA

H.-J. Kong

Department of Chemical and Biomolecular Engineering, University of Illinois Urbana, Champaign, IL, USA

M. Ericsson

Department of Cell Biology, Harvard Medical School, Cambridge, MA, USA

\section{Introduction}

Cell-interactive polymers have been widely used as synthetic extracellular matrices (sECM) to regulate cell function and promote tissue regeneration. ${ }^{[1-3]}$ Although it is known that adhesion ligand density and distribution influence the proliferation and differentiation of various cell types, ${ }^{[4,5]}$ currently available techniques do not directly characterize sECM adhesion site presentation at the nanoscale. A variety of materials in different forms, including gels and scaffolds, have been investigated as sECM to study cell-ECM adhesion ligand interactions. Tissue engineering applications of SECMs are diverse: ranging from using alginate as a SECM to promote regeneration of tissues such as bone or cartilage, ${ }^{[6,7]}$ and as a $3 \mathrm{D}$ in vitro culture system to maintain cell phenotype or promote cell maturation and differentiation. ${ }^{[8-11]}$ 
The cell attachment tripeptide sequence Arg-Gly-Asp (RGD) is commonly found in many proteins (collagen, fibronectin, vitronectin) present in the ECM, and it has been widely used to mimic ECM adhesion molecules for studying cell-ECM interactions. RGD peptides are often covalently bound to materials highly resistant to cell adhesion (e.g. alginate, ${ }^{[1,12]}$ poly(ethylene glycol) (PEG) diacrylate, ${ }^{[2]}$ poly(acrylamide $)^{[13]}$ ) such that cell adhesion is specific and precisely controlled with a high signalto-noise ratio. Furthermore, the distribution and density of adhesion ligands have been found to be important parameters regulating the adhesion, migration, proliferation and differentiation of various cell types..$^{[4,5,14]}$ As natural ECM has structural elements in the range of nanometers, nanoscale patterning of cell adhesion molecules on biomaterials has been utilized to control cell behavior. ${ }^{[15,16]} \mathrm{RGD}$ adhesion ligands presented in clusters, as opposed to a random distribution, reduced the average ligand density required to support cell migration, ${ }^{[14]}$ and greater cluster size (higher valency or number or adhesion ligands per cluster) altered actin filament organization as well. In addition, cell adhesion was observed to decrease with increasing distance between adhesion ligands. ${ }^{[17]}$ It was also observed that RGD island spacing (defined as the center-to-center distance between RGD adhesive islands) in alginate hydrogels upregulated cell proliferation, as RGD island spacing was decreased from $121 \mathrm{~nm}$ to $36 \mathrm{~nm} .{ }^{[18-20]}$ Furthermore, the proliferation of various cell types was observed to increase with greater overall RGD ligand density. ${ }^{[1,21]}$ While it is clear that RGD peptide presentation from synthetic extracellular matrices profoundly influences cell fate, there are currently no direct empirical methods of imaging RGD peptide presentation (e.g. density, distribution) from a sECMs, particularly hydrogels.

In this study, various imaging techniques including field emission scanning electron microscopy (FESEM), transmission electron microscopy (TEM) and atomic force microscopy (AFM) were employed to examine alginate hydrogels. These high resolution imaging methods were selected as the details of interest are on the nanoscale: the width of a single polysaccharide chain is on the order of $0.5 \mathrm{~nm}^{[22]}$ and the theoretical RGD island spacing in RGD nanopatterned alginate ranges from $36-121 \mathrm{~nm} \cdot{ }^{[23]}$ FESEM produces higher quality images (3-6 times better resolution) at lower voltage than conventional SEM imaging and is a more direct method of analysis, as sample preparation is minimal. For higher resolution analysis, TEM is often employed as it allows Ångström-level spatial resolution and has been used for imaging alginate hydrogel ultrastructure. ${ }^{[24,25]}$ Due to limitations of TEM, including multiple sample preparation steps, AFM has emerged as an alternative imaging technique and is particularly useful for biological samples. The AFM, invented in 1986, is widely used in nanotechnology as this method provides high resolution, 3D information under various imaging environments (e.g. air or fluid) with minimal sample preparation (e.g., fixation, staining). ${ }^{[26,27]} \mathrm{A}$ microfabricated cantilever tip, moved in the $x y z$ dimensions with a piezo translator, traces or interacts with the sample surface (in contact or tapping mode) with piconewton (pN) sensitivity. ${ }^{[28]}$ A laser is focused on the cantilever tip and reflected onto a photodiode, and resulting tip displacements are measured by the position-sensitive photodetector. An image (phase or height) with a resolution range from whole cells to single molecules ${ }^{[29]}$ is obtained by raster scanning the cantilever tip over a substrate. AFM has been employed to image a variety of soft materials including alginate, ${ }^{[22]}$ dextran, ${ }^{[30]}$ pectin $^{[31]}$ and hyaluronan. ${ }^{[32]}$ The AFM allows measurements in aqueous environments (e.g. fluid cell) which are important for biological samples, and permits determination of various biophysical properties at the molecular level such as elastic properties of single polymer strands, ${ }^{[30]}$ individual polymer conformations, ${ }^{[32]}$ and single receptor-ligand interactions. ${ }^{[33,34]}$ In addition, the AFM produces detailed 2D as well as 3D images and is a more rapid and direct method of sample characterization than EM techniques. The limitations and applications of each method for imaging alginate hydrogels are addressed in this report.

In this paper, we demonstrate a method of imaging RGD peptides covalently attached to alginate hydrogels using AFM. Streptavidin-labeled gold nanoparticles were used to label biotinylated RGD peptides covalently attached to alginate polymer chains. High resolution images at the nanoscale allow direct empirical determination of RGD distribution, and this method can be potentially applied to a variety of other biomaterials. As adhesion ligand presentation regulates cell phenotype, this method furthers our understanding of biomaterial design parameters which regulate cell fate.

\section{Experimental Part}

\section{Sample Preparation}

Peptide modified alginate was prepared using standard carbodiimide chemistry as described previously ${ }^{[12]}$ from Ultrapure medium viscous alginate gel (MVG, Pronova, Oslo, Norway) alginate and either $\mathrm{Gly}_{4}$-Arg-Gly-Asp-Ala-Ser-Ser-Lys-Biotin (G ${ }_{4}$ RGDASSK-Biotin) or $\mathrm{Gly}_{4}$-Arg-Gly-Asp-Ala-Ser-Ser-Lys (G ${ }_{4}$ RGDASSK) peptides (Commonwealth Biotechnologies, Inc., Richmond, VA). Alginates were reconstituted in alpha minimum essential media ( $\alpha$-MEM) containing $1 \%$ penicillin-streptomycin (PS) to make a $2 \%$ hydrogel solution. All chemicals were obtained from Sigma-Aldrich (St. Louis, MO) unless otherwise noted. Alginate hydrogels were ionically crosslinked using calcium sulfate slurry in a 25:1 molar ratio of calcium to alginate before casting between glass plates. Disks $(10 \mathrm{~mm}$ diameter, $1 \mathrm{~mm}$ thick) were made using an arch punch 
(McMaster-Carr, Atlanta, GA), and maintained in phenol-red free $\alpha$-MEM media with $1 \%$ PS until imaging.

\section{Gold Nanoparticle Labeling}

RGDK-biotin modified alginate hydrogel disks were labeled with streptavidin gold nanoparticles $5 \mathrm{~nm}$ in diameter (Invitrogen, Carlsbad, CA) diluted in phenol-red free $\alpha M E M / 1 \%$ PS/1\% bovine serum albumin (BSA) solution and incubated at room temperature for $2 \mathrm{~h}$. Disks were rinsed six times for 5 min each using phenol-red free $\alpha M E M / 1 \%$ PS/1\% BSA solution and maintained in phenol-red free $\alpha$-MEM media with $1 \%$ PS until imaging. RGD modified alginate (without gold labeling) were used as a negative control. To assess specificity of streptavidin gold labeling, RGDK (without biotin) modified alginate was also processed with the gold labeling procedure. This additional control was used to examine whether the gold nanoparticles bound nonspecifically to the RGD modified alginate in the absence of the biotin molecule.

\section{Alginate Imaging}

\section{FESEM}

To view the structure of alginate hydrogels, unmodified alginate disks (no RGD peptide) were fixed in ethanol, critical point freeze-dried, sputter coated with a thin layer of gold and viewed using a Quanta 200 FESEM, (FEI Inc. Co., Hillsboro, OR).

\section{TEM}

Cryo-TEM images were obtained using a JEOL 2010 FEG TEM/STEM (JEOL USA, Peabody, MA) at the Harvard Center for Nanoscale Systems Facility. RGD modified alginate disks were sucrose infiltrated and plunged into liquid ethane using a Gatan Cryoplunge. Ultrathin frozen sections were obtained using a Leica UCT ultramicrotome. Sections were deposited onto Formvar carbon-coated copper grids and viewed on a cryostage at $-190^{\circ} \mathrm{C}$.

TEM images of alginate were obtained using a JEOL 1200EX $80 \mathrm{kV}$ transmission electron microscope (JEOL USA, Peabody, MA) at the Harvard Medical School Electron Microscopy Core Facility. Ionically cross-linked RGD modified alginate disks were fixed in $4 \%$ paraformaldehyde in phosphate buffer, embedded in LR White (Electron Microscopy Sciences, Hatfield, PA), a hydrophilic acrylic embedding resin, and heat cured at $60^{\circ} \mathrm{C}$. Sections were obtained using a Reichert Ultracut $\mathrm{S}$ ultramicrotome and deposited onto Formvar carbon-coated copper grids.

The poly(vinyl alcohol) (PVA) embedding protocol was performed essentially as described by Small et al. ${ }^{[35]}$ Briefly, samples were immersed in aqueous PVA solution (20-30\% solution) for $1-2 \mathrm{~d}$ at $40^{\circ} \mathrm{C}$, dried very slowly at $40^{\circ} \mathrm{C}$, then hardened at $60^{\circ} \mathrm{C}$ within silicon mold capsules. Sections were mounted onto grids, using $87 \%$ glycerol as the flotation medium. However, the alginate samples tended to dissolve in the PVA prior to polymerization.

Epon embedding of alginate samples was as follows. Samples were fixed in $2.5 \%$ glutaraldehyde/formaldehyde solution $(1 \mathrm{~h})$, washed in sodium cacodylate buffer (30 min), post-fixed with $2 \%$ aqueous osmium tetroxide $(1 \mathrm{~h})$ and washed in sodium cacodylate buffer (30 min). Samples were dehydrated with a graduated ethanol series $(50,70,80,90,100 \%)$ and stepwise embedded in Epon 812 [25\% (1 h), 33\% (1 h), 50\% (1 h), 100\% resin $(1 \mathrm{~h})]$. Although it is reported that addition of $10 \times 10^{-3} \mathrm{M}$ calcium chloride to fixative and wash reagents prevents destabilization of alginate, ${ }^{[36]}$ those studies involved Epon embedding and sectioning of cells encapsulated in alginate. ${ }^{[36,37]}$ In our hands, alginate samples ( $2 \%$ alginate ionically cross-linked with calcium) without cells dissolved in Epon and did not section easily. Epon embedded sections are not compatible with and were not used for post-embedment gold immunolabeling. Embedding procedures were performed using fixative and wash reagents without the addition of calcium.

For gold nanoparticle labeling of RGD-biotin modified alginate hydrogels, grids containing LR White embedded sections or ultrathin frozen sections were rinsed by floating grids on drops of PBS, blocked in 1\% BSA in PBS, and incubated in solution containing $5 \mathrm{~nm}$ Alexa-488 streptavidin-labeled gold nanoparticles (Invitrogen) at room temperature for 1-2 h. Sections were rinsed with PBS, then water and allowed to dry before imaging.

AFM

AFM images were obtained at the Eastern Regional Research Center of the Agricultural Research Service (USDA) Imaging Core Facility (Wyndmoor, PA). AFM imaging of alginate was performed using a Multimode Scanning Probe microscope with a Nanoscope IIIa controller, operated as an atomic force microscope in the tapping mode (Veeco Instruments, Santa Barbara, CA) as described. ${ }^{[31]}$ Briefly, a thin layer of alginate hydrogel disks (ionically cross-linked with calcium) was 'peel-transferred' to a freshly cleaved mica surface, a method described by Fishman et al. ${ }^{[31]}$ The freshly cleaved mica was applied to the top surface of the alginate hydrogel disk, and peeled after 5-10 min. The thin alginate layer adhering to the mica surface was scanned with the AFM operating in tapping mode using etched silicon cantilevers (TESP, $20-100 \mathrm{~N} \cdot \mathrm{m}^{-1}$ spring constant, $5-10 \mathrm{~nm}$ nominal tip radius of curvature). The drive frequency, amplitude, gains, and amplitude set point ratio were adjusted to give height and phase images with the clearest image details. For gold labeled alginate samples, the gold-labeled surface (top surface of alginate hydrogel) was peel-transferred to freshly cleaved mica.

The near-neighbor spacings were determined from height images showing the gold nanoparticles superimposed on the alginate strands; the positions of the gold nanoparticles were marked on a clear transparent sheet, and the sheet was converted to calibrated digital images and analyzed using Fovea Pro 3.0 plug-ins (Reindeer Graphics, Ashville, NC) in PhotoShop 7.0 (San Jose, CA) and Microsoft Excel. This method simplified setting gray level thresholds that accurately identified only the gold nanoparticles and excluded all regions of the alginate strands.

\section{Results and Discussion}

\section{EM Imaging of Alginate Hydrogels}

The nanoscale characteristics (architecture, porosity, RGD ligand distribution) of alginate hydrogels were examined using high resolution imaging techniques as the details of 
interest are on the nanoscale. FESEM was the first method attempted for image analysis, as sample preparation is minimal. As wet samples are not suitable for imaging under the high vacuum conditions for FESEM, alginate hydrogels were dehydrated and given an electroconductive

a)

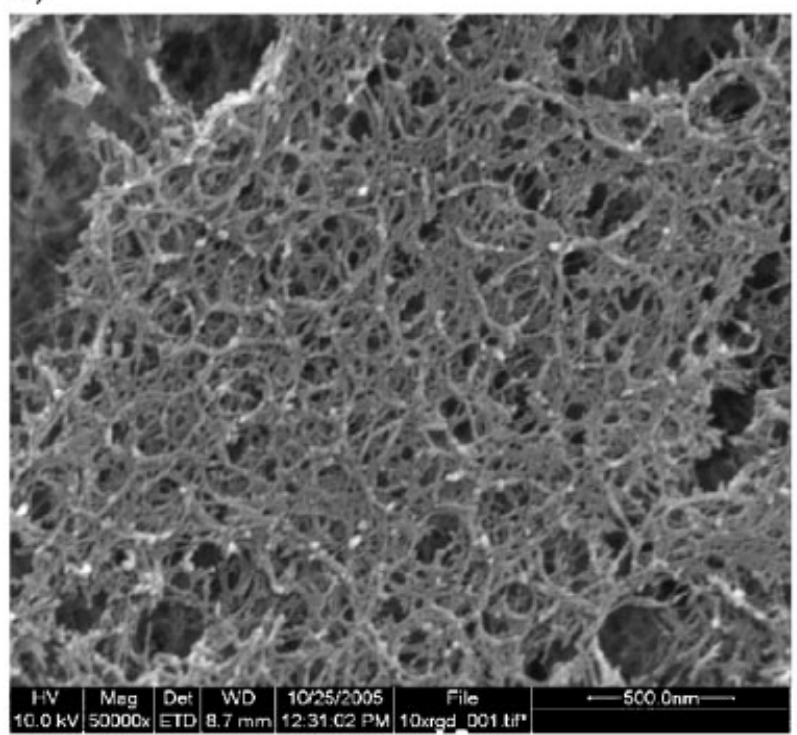

b)

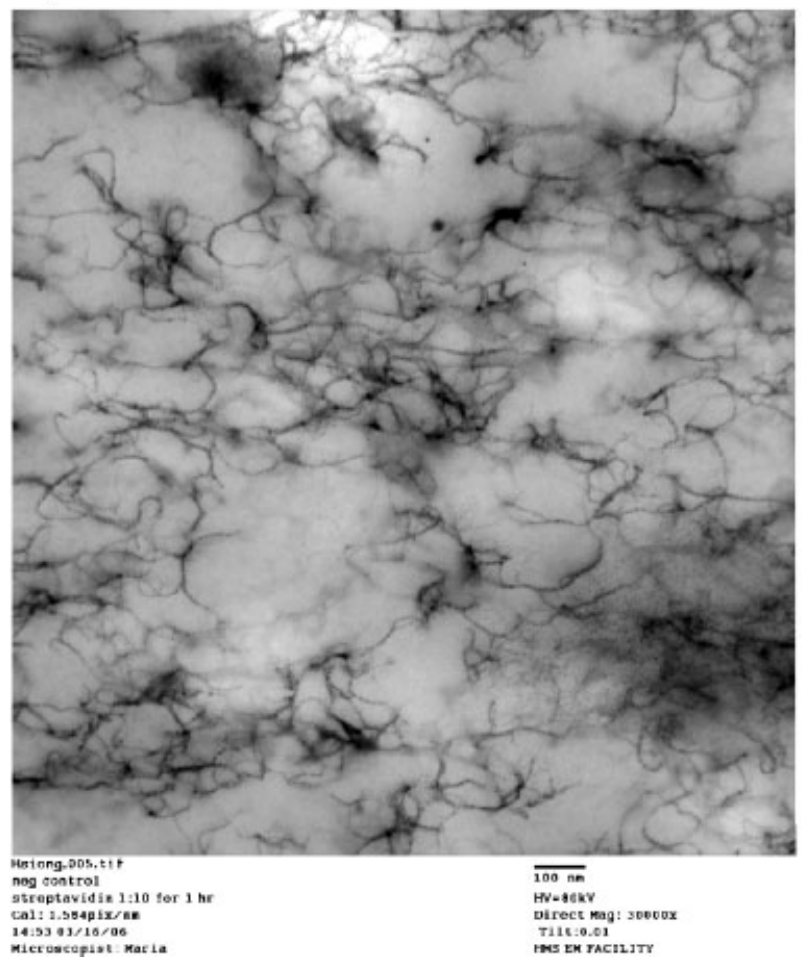

coating (e.g., gold). However, the dehydration step and critical point freeze drying often induced cracks in the sample due to shrinkage, as observed in the FESEM image of the alginate hydrogel (Figure 1a), and the gold coating can obscure the details of interest. Although FESEM

c)

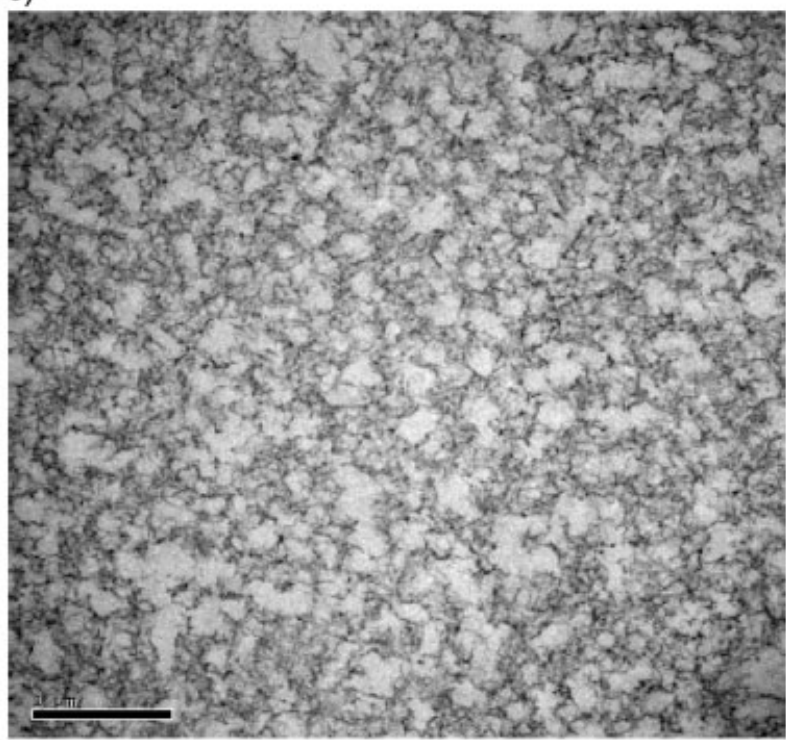

d)

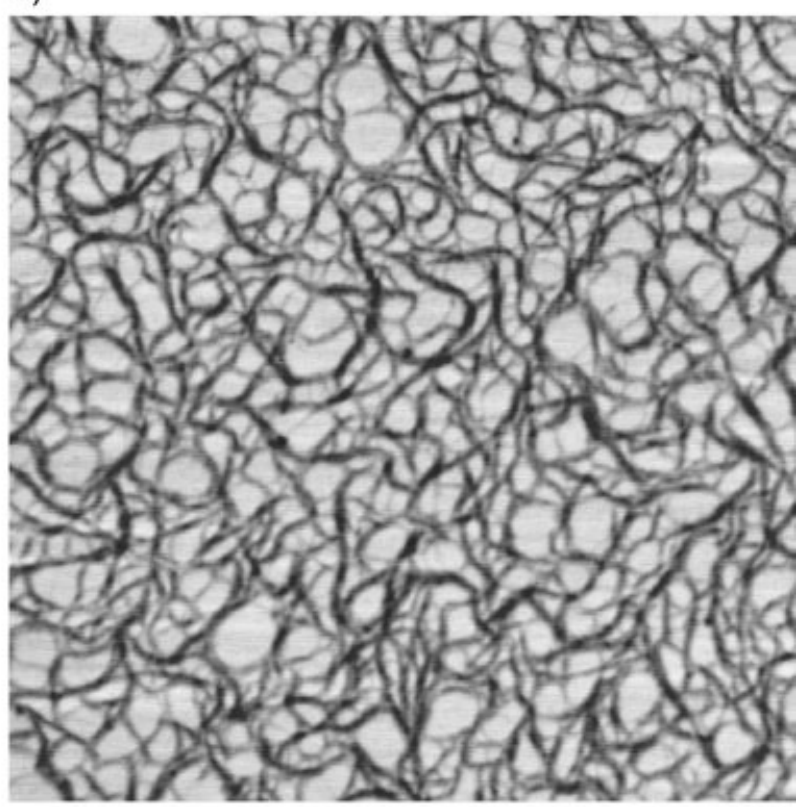


provides a quick and broad examination of the sample, the resolution was insufficient. For higher resolution analysis, TEM imaging was conducted. FESEM (Figure 1a) and TEM (Figure 1b) images demonstrate that alginate hydrogels are a mesh-like, fibrous networks with a nanosized pore structure, as expected. TEM imaging of alginate samples was challenging, as the sample structure was often compromised during the embedding step and portions of the sample would disintegrate, regardless of the embedding media tested (LR White, PVA, Epon 812). EM imaging at the high voltages required for high resolution also produced sample damage due to prolonged sample exposure to the electron beam. Cryo-TEM was subsequently used as this low-temperature technique reduces sample damage at high voltages while also eliminating the embedding step. Cryo-TEM images of alginate hydrogel disk sections showed a dense polymer network with nanosized pores (Figure 1c). Analysis of the TEM image (Figure 1c) reveals that the large pores observed throughout the alginate sample had an average pore size of approximately $150 \mathrm{~nm}$. These large pores may be due to the presence of ice crystals formed within the alginate sample during the freezing process. Analysis of the smaller pores within the dense polymer network of the sample would yield pore sizes closer to previously reported values (few nanometers). ${ }^{[39]}$ FESEM and cryo-TEM images were similar to those previously published for cryo-SEM and TEM images of alginate beads, respectively. ${ }^{[24,25]}$ However, the disadvantage of these methods is the requirement for extensive sample processing, which may introduce artifacts.

\section{AFM Imaging of Alginate Hydrogels}

AFM was subsequently used as an alternative technique, as this imaging method allows high resolution imaging with minimal sample processing. Initial imaging of the alginate hydrogel samples was unsuccessful due to the "stickiness" of the hydrogel, which interfered with the AFM cantilever tip. To circumvent this issue, a previously described method for AFM imaging of pectin gels was employed. ${ }^{[31]}$ Freshly cleaved mica was applied to the surface of an alginate hydrogel disk sample and peeled. A thin alginate hydrogel layer peel-transferred to the mica was imaged in the tapping mode in air. Height and phase shift images of alginate samples reflect the surface topography and adhesive regions of the sample, respectively. Images demonstrated a network-like structure with irregular pore sizes (Figure $1 \mathrm{~d}, 2.5 \mu \mathrm{m}$ scale) similar to that visualized for other polysaccharides. ${ }^{[38]}$ The gels consisted of branched structures where the branch points may be interpreted as points where cooperative junctions between calcium cross-linked alginate chains occur, similar to what is observed in pectin sugar gels. ${ }^{[31]}$ The nanosized pores range from approximately $10-100 \mathrm{~nm}$, with the average pore diameter closer to $40 \mathrm{~nm}$. Some of the pores in Figure $1 \mathrm{~d}$ were divided by a secondary network, perhaps due to subsurface alginate layers. The average pore size distribution in alginate gels as determined by a thermoporometry technique has been reported on the order of 5-6 nm. ${ }^{[39]}$ The discrepancy between the previously reported value and that obtained from AFM images may be due to incomplete transfer of the alginate gel layer to the mica, and highlights the importance of consistent sample preparation for analysis. In the AFM images, the height measurements of individual polymer fibers were approximately $0.5 \mathrm{~nm}$, as expected, supporting the assumption that approximately one polymer layer was peel-transferred to the mica. The lateral width of single polysaccharide chains is usually reported to be around $0.5 \mathrm{~nm},{ }^{[22]}$ and the greater width of polymer fibers measured via AFM may be due to aggregates of individual alginate chains or tip broadening effects ${ }^{[40]}$ where the tip geometry (radius of curvature) causes overestimation of the molecular dimension being measured in the $x y$ plane.

\section{Gold Nanoparticle Labeling of Adhesion Ligands}

Adhesion ligand sequences are only several nanometers in length and are indistinguishable from the polymer matrix at resolutions available from imaging these types of soft materials. A novel method was developed to enable peptide detection by using streptavidin-bound gold nanoparticles to label biotinylated RGD peptides in the alginate hydrogel (Figure 2). Specifically, streptavidin bound colloidal gold nanoparticles (5 $\mathrm{nm}$ in diameter) were selected for peptide labeling as these particles are sufficiently small for high resolution and yet are large enough to remain distinguishable from the polymer background (e.g. via height differences measured from AFM).

RGD ligand distribution in the alginate hydrogel was imaged using both TEM and AFM, complementary techniques for high resolution imaging. TEM images of alginate sections (embedded in LR White and post-labeled with gold nanoparticles) showed minimal gold binding (Figure 3). Neither elemental analysis using X-ray photoelectron spectroscopy (XPS) nor secondary electron backscatter detected gold in the samples (data not shown). The alginate tended to dissolve during the curing step, and other preparation steps for EM imaging (e.g. dehydration, fixation and staining) may have compromised the sample. Alginate hydrogel samples were also pre-labeled with gold nanoparticles (both surfaces incubated with gold nanoparticles), and prepared for sectioning and EM imaging. However, it was extremely difficult to obtain ultrathin 


\section{RGD modified alginate hydrogel disk}
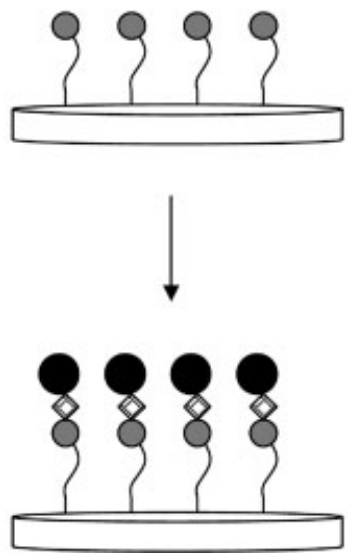

Streptavidin conjugated gold nanospheres $(5 \mathrm{~nm})$

\author{
RGD-Biotin Peptide
}

Figure 2. Schematic of technique to label RGD modified alginate hydrogels with streptavidin-gold nanoparticles ( $5 \mathrm{~nm}$ ), for imaging RGD distribution in alginate hydrogels. RGD modified alginate hydrogel disks (ionically cross-linked with calcium) are incubated in streptavidin gold nanoparticle solution. Streptavidin-labeled gold nanoparticles bind to biotinylated RGD sequences and label RGD cell adhesion molecules.

sections of only the top surface (gold labeled) of the alginate hydrogels. Cryo-TEM of ultrathin sections postlabeled with gold nanoparticles was also unsuccessful (data not shown). Although the alginate structure was better maintained, gold binding was minimal with this

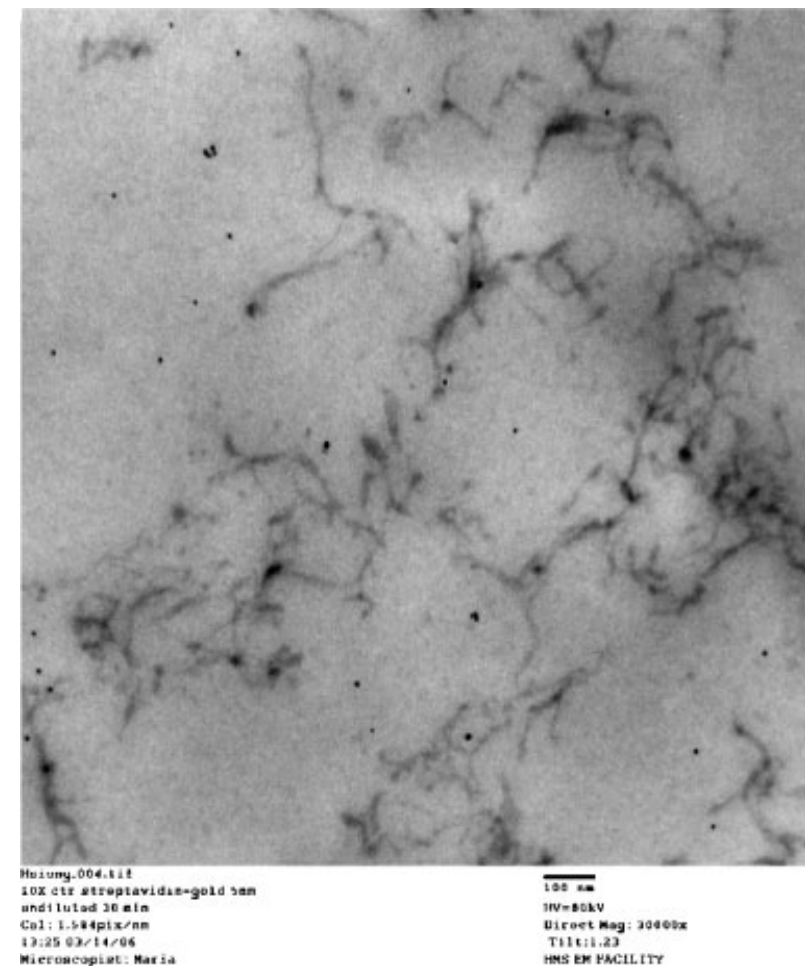

Figure 3. TEM images of RGD modified alginate disks, (with gold nanoparticle labeling). Alginate hydrogel disks were fixed in $4 \%$ paraformaldehyde, embedded in LR White, sectioned and postlabeled with $5 \mathrm{~nm}$ streptavidin gold nanoparticles. Scale bar is $100 \mathrm{~nm}$. latter approach. The minimal gold binding to RGD modified alginate may result from damage to the gel structure and/ or RGD peptide during sample processing for EM. It is possible that further optimization of TEM imaging can also yield good quality images but may require improved contrast agents or embedding protocols. ${ }^{[41]}$

RGD ligand distribution in alginate hydrogels was subsequently imaged using AFM where the top surface of gold labeled RGD modified alginate samples was peel transferred to mica for AFM imaging. AFM images of gold labeled RGD modified alginate (two peptides per alginate chain or degree of substitution (DS) of 2) indicated a uniform distribution of gold nanoparticles throughout the alginate surface (Figure 4a). The height of gold nanoparticles bound to alginate chains of the polymer network was approximately $5.5 \mathrm{~nm}$ (analyzed from the images using the Nanoscope software version $5.12 \mathrm{rev}$ B as supplied by the manufacturer) (Figure $4 \mathrm{~b}$ ), the total expected height of a $5 \mathrm{~nm}$ gold nanoparticle and $0.5 \mathrm{~nm}$ polysaccharide monomer. The average nearest neighbor distances (obtained from image processing and analysis) between gold particles (reflecting RGD adhesion ligand spacing) was $21 \mathrm{~nm}$ (Figure 4c), which is similar in magnitude to the theoretical distance of $36 \mathrm{~nm} \cdot{ }^{[18]}$ Image analysis also indicates a broad distribution of nearest neighbor distances. Evaluation of a separate image obtained from a different sample field yielded similar values for nearest neighbor spacing (data not shown). The average nearest neighbor distance obtained underestimates the spacing between RGD adhesion ligands and the calculation of the average distance between a gold nanoparticle and its six nearest neighbors may perhaps more accurately reflect the overall adhesion ligand spacing. As shown in Figure 4d, gold nanoparticle labeling 
a)

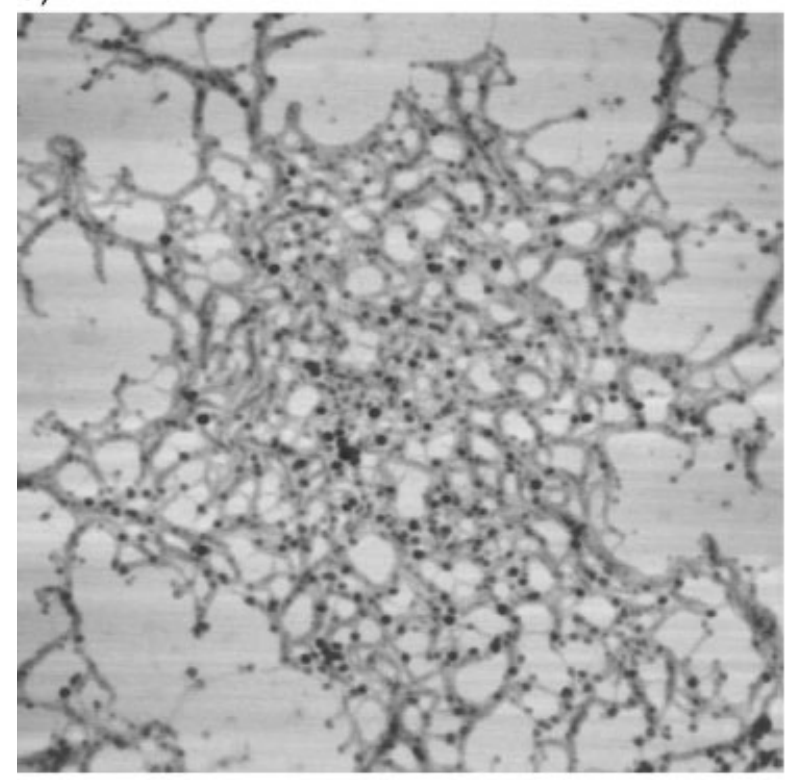

b)
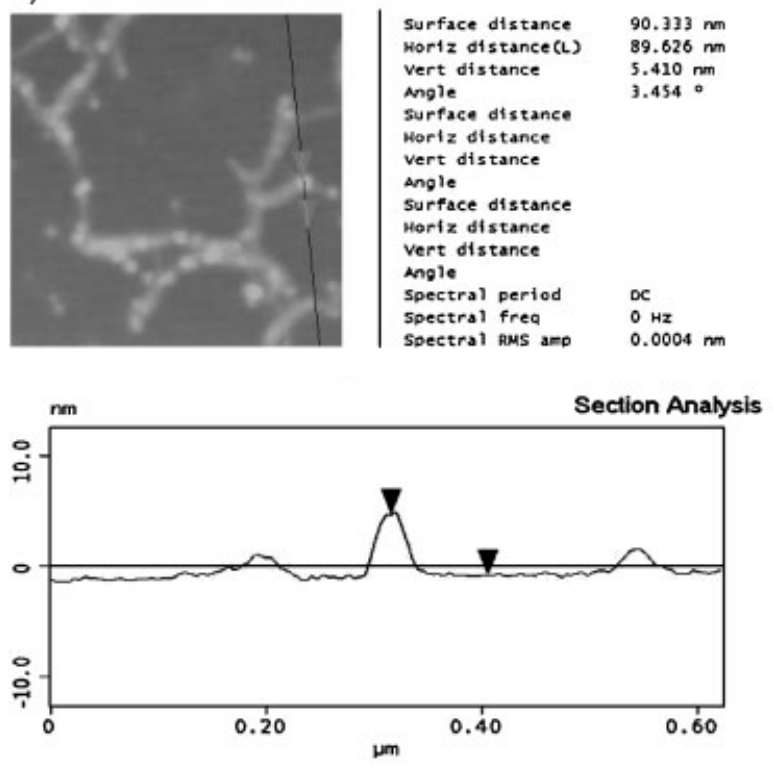

c)

\section{Particle Near-Neighbor Spacings}

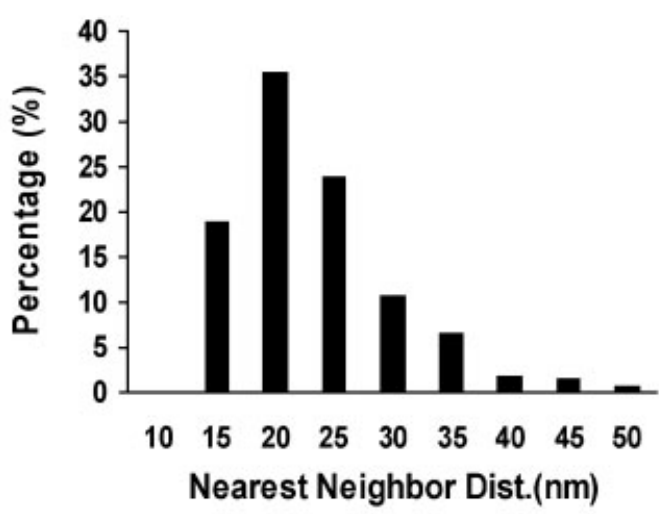

d)

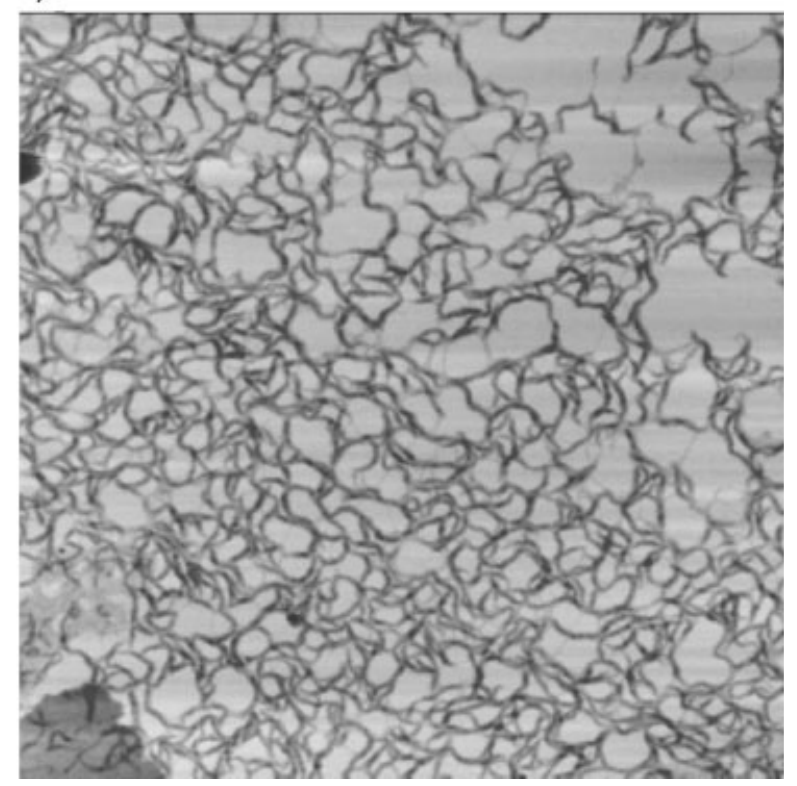

Figure 4. a) AFM image of thin RGD modified alginate hydrogel layer (DS 2 RGDK-biotin modified alginate, labeled with $5 \mathrm{~nm}$ streptavidin gold nanoparticles), peel-transferred to freshly cleaved mica surface. Scale is $2.5 \mu \mathrm{m}$. b) Height analysis of dots along alginate polymer in image reveals dimensions of approximately $5 \mathrm{~nm}$ in height, confirming that these are $5 \mathrm{~nm}$ gold nanoparticles. c) Nearest-neighbor distance between gold nanoparticles (obtained from Figure 4a), reflecting spacing between RGD adhesion ligands. Average distance between nanoparticles are $21 \mathrm{~nm}$. d) AFM image of thin RGD modified alginate hydrogel layer (DS 2 RGDK modified alginate, labeled with $5 \mathrm{~nm}$ streptavidin gold nanoparticles), peel-transferred to freshly cleaved mica surface. Scale is $2.5 \mu \mathrm{m}$. Images were obtained using an AFM with a Nanoscope Illa controller (Veeco) operated in tapping mode.

of RGDK (no biotin) modified alginate hydrogel disks did not result in gold binding to the alginate sample, indicating that streptavidin gold nanoparticles were specifically bound to biotinylated RGD modified alginate only.
This AFM imaging technique can be extended to examine different ligand densities presented from alginate hydrogels or other materials used as sECMs. Presentation of adhesion ligands in clusters are often of interest as ligand clustering and ligand spacing are parameters 
known to influence cell behavior. ${ }^{[14,42,43]}$ Determination of ligand distribution (presented uniformly or in clusters) can be similarly performed using gold nanoparticle labeling and subsequent AFM imaging, as long as the distance between clusters is of greater magnitude than the distance within clusters. However, as each nanoparticle is associated with several (approximately 1-2) streptavidin molecules, there is a possibility of a single nanoparticle cross-linking adjacent biotinylated adhesion ligands. Also, utilization of the $5 \mathrm{~nm}$ streptavidin labeled gold nanoparticles may be limited in determining clusters of adhesion ligands in close proximity, due to the size of the gold particles. This limitation may be circumvented by employing smaller gold nanoparticles, such as the $1.4 \mathrm{~nm}$ nanogold streptavidin product (Nanoprobes, Yaphank, NY) in which a single streptavidin molecule is covalently conjugated to a single gold particle. The drawback of using the smaller gold nanoparticles is that their detection against the polymer strands becomes challenging.

Of the various imaging methods utilized to examine alginate hydrogel structure and RGD ligand distribution, the AFM technique described here is a relatively quick and direct method which yields high resolution images with minimal sample preparation. Although further optimization of EM imaging or other techniques may yield similar results, these methods often require multiple processing steps.

\section{Conclusion}

A novel method to view RGD ligand presentation from alginate hydrogels was developed by using streptavidingold nanoparticles to label biotinylated peptides. AFM imaging of thin alginate hydrogel layers peel-transferred to freshly cleaved mica was the method of choice for obtaining high resolution images of alginate hydrogels. AFM images of RGD modified alginates allowed qualitative determination of the specificity of RGD coupling to alginate polymer chains, as well as RGD ligand distribution.

Although streptavidin labeled colloidal gold was used to tag RGD ligands, other streptavidin labeled nanoparticles such as quantum dots could also be used. In the future, quantitative analysis of RGD ligand distribution could be employed using software to parse the gold nanoparticles from the alginate background, and to calculate average distances between RGD ligands. In summary, this data indicates that high resolution imaging techniques can be powerful tools for identifying and understanding important physical properties of biomaterials and cell adhesion ligand distribution. As nanomaterials are increasingly utilized in biomedical technologies, ${ }^{[4]}$ AFM and other analytical tools will play a critical role in enhancing our understanding of cell-biomaterial interactions.
Acknowledgements: This work was supported by the U.S. Army Research Laboratory and the U.S. Army Research Office grant DAAD190310168. We thank Dr. Richard Schalek and Dr. David Bell from the Harvard Center for Nanoscale Systems (CNS) for their help with Cryo-TEM imaging. Harvard CNS is a member of the National Nanotechnology Infrastructure Network (NNIN), which is supported by the National Science Foundation under NSF award no. ECS-0335765. CNS is part of the Faculty of Arts and Sciences at Harvard University.

The authors declare no competing financial interests.

Received: November 30, 2007 Revised: February 2, 2008; Accepted: February 8, 2008; DOI: 10.1002/mabi.200700313

Keywords: atomic force microscopy (AFM); biomaterials; electron microscopy; hydrogels; TEM

[1] E. Alsberg, K. W. Anderson, A. Albeiruti, R. T. Franceschi, D. J. Mooney, J. Dent. Res. 2001, 80, 2025.

[2] D. L. Hern, J. A. Hubbell, J. Biomed. Mater. Res. 1998, 39, 266.

[3] E. Hill, T. Boontheekul, D. J. Mooney, Proc. Natl. Acad. Sci. U. S. A. 2006, 103, 2494.

[4] D. Harrison, R. Johnson, M. Tucci, A. Puckett, A. Tsao, J. Hughes, H. Benghuzzi, Biomed. Sci. Instrum. 1997, 34, 41.

[5] A. Rezania, K. E. Healy, J. Biomed. Mater. Res. 2000, 52, 595.

[6] E. Alsberg, K. W. Anderson, A. Albeiruti, J. A. Rowley, D. J. Mooney, Proc. Natl. Acad. Sci. U. S. A. 2002, 99, 12025.

[7] C. M. Mierisch, H. A. Wilson, M. A. Turner, T. A. Milbrandt, L. Berthoux, M. L. Hammarskjold, D. Rekosh, G. Balian, D. R. Diduch, J. Bone Jt. Surg., Am. Vol. 2003, 85A, 1757.

[8] Y. Park, M. Sugimoto, A. Watrin, M. Chiquet, E. B. Hunziker, Osteoarth. Cart. 2005, 13, 527.

[9] H. Tanaka, C. L. Murphy, C. Murphy, M. Kimura, S. Kawai, J. M. Polak, J. Cell. Biochem. 2004, 93, 454.

[10] D. Dufrane, R. M. Goebbels, A. Saliez, Y. Guiot, P. Gianello, Transplantation 2006, 81, 1345.

[11] P. K. Kreeger, J. W. Deck, T. K. Woodruff, L. D. Shea, Biomaterials 2006, 27, 714.

[12] J. A. Rowley, G. Madlambayan, D. J. Mooney, Biomaterials 1999, 20, 45.

[13] B. K. Brandley, R. L. Schnaar, Anal. Biochem. 1988, 172, 270.

[14] G. Maheshwari, G. Brown, D. A. Lauffenburger, A. Wells, L. G. Griffith, J. Cell Sci. 2000, 113, 1677.

[15] M. Shin, H. Yoshimoto, J. P. Vacanti, Tissue Eng. 2004, 10, 33.

[16] C. Y. Xu, R. Inai, M. Kotaki, S. Ramakrishna, Biomaterials 2004, 25, 877.

[17] Y. N. Danilov, R. L. Juliano, Exp. Cell Res. 1989, 182, 186.

[18] K. Y. Lee, E. Alsberg, S. Hsiong, W. Comisar, J. Linderman, R. Ziff, D. J. Mooney, Nano Lett. 2004, 4, 1501.

[19] W. A. Comisar, S. X. Hsiong, H. J. Kong, D. J. Mooney, J. J. Linderman, Biomaterials 2006, 27, 2322.

[20] L. Y. Koo, D. J. Irvine, A. M. Mayes, D. A. Lauffenburger, L. G. Griffith, J. Cell Sci. 2002, 115, 1423.

[21] J. A. Rowley, D. J. Mooney, J. Biomed. Mater. Res. 2002, 60, 217.

[22] A. Decho, Carbohydr. Res. 1999, 315, 330.

[23] K. Y. Lee, E. Alsberg, S. X. Hsiong, W. A. Comisar, J. J. Linderman, R. Ziff, D. J. Mooney, Nano Lett. 2004, 4, 1501.

[24] D. Serp, M. Mueller, U. Von Stockar, I. W. Marison, Biotechnol. Bioeng. 2002, 79, 243.

[25] D. Serp, M. Mueller, U. Von Stockar, I. W. Marison, Biotechnol. Bioeng. 2002, 79, 253. 
[26] N. C. Santos, M. A. Castanho, Biophys. Chem. 2004, 107, 133.

[27] G. Binnig, C. F. Quate, C. Gerber, Phys. Rev. Lett. 1986, 56, 930.

[28] V. Dupres, C. Verbelen, Y. F. Dufrene, Biomaterials 2007, 28 , 2393.

[29] Z. Shao, J. Yang, A. P. Somlyo, Annu. Rev. Cell Dev. Biol. 1995 , 11, 241.

[30] M. Rief, F. Oesterhelt, B. Heymann, H. E. Gaub, Science 1997, 275, 1295

[31] M. Fishman, P. Cooke, D. Coffin, Biomacromolecules 2004, 5 , 334.

[32] C. Spagnoli, A. Korniakov, A. Ulman, E. A. Balazs, Y. L. Lyubchenko, M. K. Cowman, Carbohydr. Res. 2005, 340, 929.

[33] C. M. Franz, A. Taubenberger, P. H. Puech, D. J. Muller, Sci. STKE 2007, 2007, p. 15.

[34] H. Yang, J. Yu, G. Fu, X. Shi, L. Xiao, Y. Chen, X. Fang, C. He, Exp. Cell Res. 2007, 313, 3497.
[35] J. V. Small, D. O. Furst, J. De Mey, J. Cell Biol. 1986, 102, 210.

[36] M. Wong, M. Siegrist, V. Gaschen, Y. Park, W. Graber, D. Studer, Tissue Eng. 2002, 8, 979.

[37] M. B. Evangelista, S. X. Hsiong, R. Fernandes, P. Sampaio, H. J. Kong, C. C. Barrias, R. Salema, M. A. Barbosa, D. J. Mooney, P. L. Granja, Biomaterials 2007, 28, 3644.

[38] M. Fishman, P. Cooke, H. Chau, D. Coffin, A. Hotchkiss, Biomacromolecules 2007, 8, 573.

[39] T. Boontheekul, H. J. Kong, D. J. Mooney, Biomaterials 2005 26,2455

[40] V. J. Morris, A. R. Kirby, A. P. Gunning, "Atomic Force Microscopy for Biologists", 1st edition, Imperial College press, London 2004.

[41] S. Reese, B. Guggenheim, Microsci. Res. Technol. 2007, 70, 816.

[42] S. P. Massia, J. A. Hubbell, J. Cell Biol. 1991, 114, 1089.

[43] E. A. Cavalcanti-Adam, T. Volberg, A. Micoulet, H. Kessler, B. Geiger, J. P. Spatz, Biophys. J. 2007, 92, 2964.

[44] H. Liu, T. J. Webster, Biomaterials 2006, 28, 354. 